\title{
The lack of UME6 sustain 2- deoxy-D-glucose toxicity under inositol limitation condition in Saccharomyces cerevisiae
}

\author{
Chidambaram Ravi ${ }^{1}$ and Vasanthi Nachiappan ${ }^{2} *$ \\ ${ }^{1,2}$ Department of Biochemistry, School of Life Sciences, Bharathidasan University, Tiruchirappalli, India \\ * Corresponding author-vasanthibch@gmail.com, Mobile: +918973721898,
}

Available online at: www.isroset.org

Received: 11/Feb/2019, Accepted: 22/Feb/2019, Online: 28/Feb/2019

\begin{abstract}
The glucose analogues 2-DG exposure affects glycolytic enzymes. Glucose-6 P is the precursor for the synthesis of myo-inositol metabolism, 2-DG exposer reduced myo-inositol level. In the current study, we found UME6 deletion depict resistance to the 2-DG toxicity. The UME6 deletion increased the INO1 $\beta$-galactosidase activity even the exposer of 2-DG in $(0.025 \%$ and $0.05 \%)$ under I- condition compared to WT cells. The sever growth impairment was observed in $0.025 \%$ concentration of 2-DG, and also severally affects the INOI transcription in WT cells under inositol deprivation. The $0.05 \%$ concentration highly toxic no growth was observed under inositol limitation, which led to abolish the INOI transcription in WT cells. The UME6 transcription factor negatively regulates $I N O 1$ transcription this could be the reason for the resistance ume6 $\Delta$ strain of 2-DG toxicity with I- condition. We conclude that the ume6s resistant with the exposure of 2-DG under inositol limitation condition in Saccharomyces cerevisiae.
\end{abstract}

Keywords- 2-deoxy-D-glucose, INO1, $\beta$-Galactosidase activity, over production of inositol.

\section{INTRODUCTION}

Yeast UME6 gene encode unscheduled meiotic gene expression which represses transcription of a diverse set of genes involved in meiosis (1), heat shock response and arginine catabolism $(1,2)$. The Ume6p also a positive regulator of some early meiotic genes in sporulating cells $(1$, 2). However, Ume6p also positively regulates the CDP-DAG pathway genes $\mathrm{CHO}, \mathrm{CHO} 2$ and $\mathrm{OPI} 3$ but negatively regulates $I N O 1$ as well as the phospholipid biosynthetic genes in yeast (2).

Glucose-6-P is the precursor for carbohydrate storage metabolism (trehalose, glycogen) and also inositol metabolism (4).The INOI encode inositol-3-P synthase catalyzed the conversion of Glucoe-6 P to inositol-3 P (3). Previous report suggest that the 2-deoxy-D-glucose (2-DG) is a well-known glucose analogue and the exposure leads to defective glycolysis (5). The 2-DG exposure causes multiple defects growth impairment, cell morphology, cell wall biosynthesis, and cell lysis (4). Glucose-6 P procure from glucose with the help of $(H X K)$.

The hexokinase also catalyzed the conversion of glucose analogue 2-DG to 2-DG-6 P, that toxic substance cannot metabolized $(6,7)$.Inositol is an essential metabolites modulates a diverse cellular process including apoptosis (8), membrane trafficking (9), and are involved in glycolipid and phospholipid metabolism as well (10). Hence, inositol level also defect under 2-DG exposure in mice (7).

The deletion of UME6 displays over production of inositol (Opi- phenotype) and negatively regulates INOI transcription (2). The INOI transcription was positively regulated by Ino2-Ino4 heterodimer complex, Opilp repress the INOI under inositol presence condition $(8,9)$. Similarly, previous study also depict deletion of OPII rescue the sensitivity of 2-DG under inositol deprivation condition (10). The deletion of GCRl also depict highly sensitive with the exposure of 2-DG under normal condition (10).

In this work we established the importance of meiotic regulating transcription factor ume6 6 under 2-DG sensitivity in Saccharomyces cerevisiae. Additionally, we examine the INOI-LacZ activity to exposure of glucose analogue (2-DG) in WT and ume6s either presence or absence of inositol and 2-DG $(0.025 \%$ and $0.05 \%)$. The current study depict the ume6s (Opi- phenotype) strains rescue the 2-DG toxicity under inositol deprivation condition.

\section{MATERIALS AND METHODS}

Media, strains and growth condition.

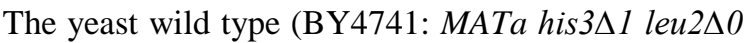
met $15 \Delta 0$ ura $3 \Delta 0)$ and ume $6 \Delta$ strains were gifted by Prof. Ram Rajasekharan, Central Food Technological Research 
Institute (CFTRI), Mysore, India. Yeast strains was grown in YPD (1\% in yeast extract, $2 \%$ peptone, and $2 \%$ dextrose) medium ( $\mathrm{pH} 7.0$ ) or synthetic complete/ defined media contains yeast nitrogenous base $0.67 \mathrm{~g}$ (YNB), yeast dropout with respective amino acids $(0.14 \mathrm{~g}$ uracil $7.6 \mathrm{mg}$, tryptophan $7.6 \mathrm{mg}$, leucine $38 \mathrm{mg}$, and histidine $7.6 \mathrm{mg}$ ) and $100 \mathrm{mg} / \mathrm{L}$ kanamycin with aeration at $30{ }^{\circ} \mathrm{C}$ at $180 \mathrm{rpm}$ for $24 \mathrm{~h}$. The cell growth was monitored by measuring the absorbance at $600 \mathrm{~nm}$. The YEp357R vector containing INO1-LacZ was gifted from John M. Lopes, College of Natural Sciences, University of Massachusetts, Amherst, MA. The yeast INO1LacZ containing YEp357R vector was used for promoter reporter analysis. Cells were grown until mid-log phase in 5 $\mathrm{mL}$ of SD-U containing $2 \%$ glucose. Then the cells were pelleted and resuspend with fresh SD-U medium containing presence (I+) or absence (I-) of inositol cells were continue shaking with $180 \mathrm{rpm}$ at $30^{\circ} \mathrm{C}$. The $E$. coli transformants was selected on LBA plates containing $0.5 \%$ yeast extract, $1 \%$ peptone, $1 \% \mathrm{NaCl}$, and $100 \mathrm{mg} / \mathrm{L}$ ampicillin.

Cell tolerance assessment of 2-Deoxyglucose (2-DG) resistance by plate assay and growth curve analysis.

The overnight culture of WT and ume $6 \Delta$ cells were grown in YPD media at $30{ }^{\circ} \mathrm{C}$. The cells were equalized by OD 600 and equal number of cells were serially diluted (10fold) with autoclaved double distilled water, and $3 \mu$ l aliquots of each dilution were spotted onto SC and the media containing either presence or absence of inositol with and without exposure of $0.025 \%$ and $0.050 \%$ of $2-\mathrm{DG}$ and $2 \%$ dextrose as a carbon source containing agar plates and incubated $30{ }^{\circ} \mathrm{C}$ for 3 days. The growth curve analysis cell growth was monitored by measuring the absorbance $\left(\mathrm{A}_{600 \mathrm{~nm}}\right)$ of the cell density at indicated times points until $64 \mathrm{~h}$.

\section{$\beta$-Galactosidase activity of INO-LacZ}

Wild-type and ume6 $\Delta$ strains were transformed with YEp357-INO1-LacZ fusion gene containing plasmid and the transformants were grown in to SD-U (synthetic defined uracil) media up to mid-log phase at $30{ }^{\circ} \mathrm{C}$. Then the cells were washed with fresh SD-U media and cells were subcultures in to SD-U containing either I- or I+, and the presence or absence of $0.025 \%$ and $0.050 \%$ of $2-\mathrm{DG}$ and incubated at $30{ }^{\circ} \mathrm{C}$ for $4 \mathrm{~h}$. The cell free extract were collected and extracted protein was quantified by Bradford method [13] and measured the $\beta$-Galactosidase activity as described previous method of Rose and Botstein [14]. The INO1-LacZ activity was expressed nmol. $\mathrm{min}^{-1} . \mathrm{mg}^{-1}$ protein.

\section{Statistical analysis}

The experimental data quantification was examined by using Student's t-test, and the obtained variance were considered statistically significant $* \mathrm{p}<0.05$. Respectively at least three independent experiments was repeated. Data are presented as the average \pm standard deviation (SD). Statistical examination was performed using the sigma plot 10.0 software.

\section{RESULTS AND DISCUSSION}

Growth assessment and INO1- LacZ activity in WT and ume6 $\Delta$ strain under both presence and absence of inositol.

In the present study, we examine the growth using spot assay and growth curve analysis in WT and ume $6 \Delta$ strain under inositol limitation ( $\mathrm{I}-$ ) and inositol supplementation. We found an increases the growth Inositol is an important for the cell growth (10), the lack of inositol reduces the growth in WT cells (Fig. 1B).

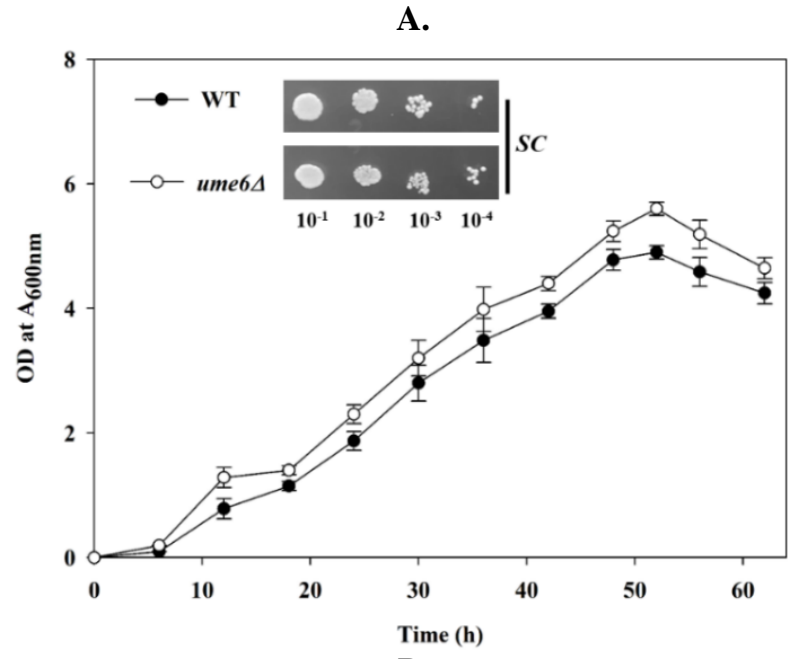

B.

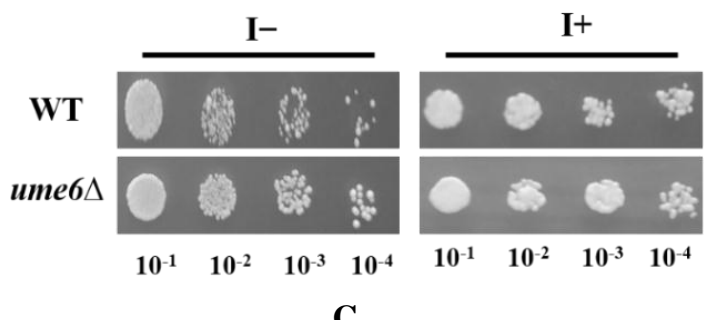

C.

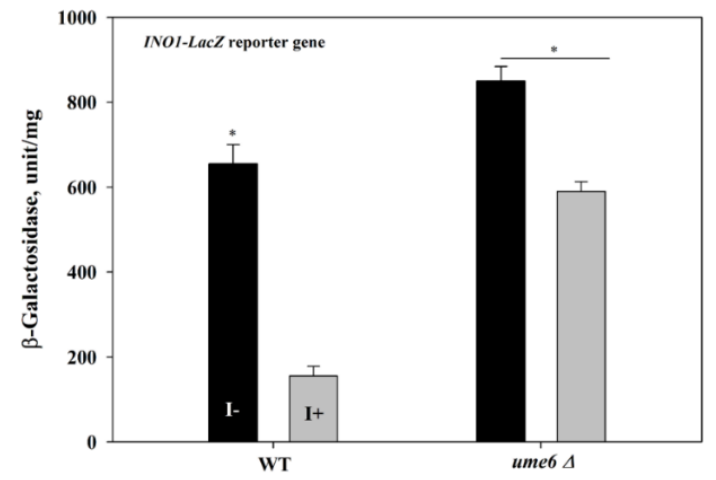

Fig.1 Effect of growth under presence and absence of inositol and INO1- LacZ activity in WT and ume6 $\triangle$. 
The WT and ume6s strain were cultured in YPD media and grown up to mid-log phase at $30{ }^{\circ} \mathrm{C}$. (A). The growth curve was monitored by measuring $\mathrm{OD}\left(\mathrm{A}_{600 \mathrm{~nm}}\right)$ of the cells at indicate time points until $64 \mathrm{~h}$. The growth curve is an average of three experimental repeats. The cells were serially diluted (tenfold), and $3 \mu \mathrm{l}$ of cells were spotted onto SC $2 \%$ dextrose containing agar plates and incubated for 3 days at 30 ${ }^{\circ} \mathrm{C}$ (Insert image spot test). (B). The mid log phase grown cells were serially diluted, and $3 \mu$ l of cells were spotted onto SC $2 \%$ dextrose with the presence or absence of inositol containing agar plates and incubated for 3 days at $30^{\circ} \mathrm{C}$. (C). $\beta$-galactosidase activity. The cell harbouring YEp357R plasmid containing INOI-LacZ fusion gene containing WT cells were cultured in SD-Ura. The mid-log phase grown cells were washed with $\mathrm{I}-$ free media and cells were seeded into both presence and absence of inositol. The cell extract were subjected to measure the $\beta$-Galactosidase activity. The specific $\beta$-Galactosidase activity was expressed as units mg-1 (nmol min-1 mg-1). The data shown is average of three independent experiments $(* \mathrm{P}<0.05)$.

The UME6 is a negative regulator of INOI transcription (2), hence the deletion of UME6 also shown to increases the growth under I- (Fig. 1B). Normally, INOI highly expressed under I- condition $(8,9)$. The INOI promoter fused with $\mathrm{LacZ}$ gene also increased $(\sim 28 \%)$ under I- and in ume $6 \Delta$ compared to WT I- cells. The activity of $\beta$-galactosidase still increased under I+ in ume6 $\Delta$ (Fig. 1C), which indicates the deletion of UME6 increased the growth under I- condition compared to WT cells.

Effect of $0.025 \%$ 2-DG on INO1-LacZ activity in the presence and absence of inositol.

The lack of Ume6p display Opi-phenotype and increased the INO1-LacZ expression (Fig.1). Further we established the $\beta$-galactosidase activity of INOI under I- and I+ with the exposure of $0.025 \%$ 2-DG. Because the presence of 2-DG leads to increases a toxic substance 2-DG phosphate, which cause on inhibitory effect on myo-inositol-1-phosphate production (11). The deletion of UME6 cells with the $0.025 \%$ 2-DG exposure increased the activity of INO1-LacZ under Iand I+ when compared to WT cells (Fig.2 A), but reduced the expression of INO1-LacZ compared to untreated ume $6 \Delta$ cells (Fig.1C).

Growth assessment of $0.025 \%$ 2-DG on plate assay and growth curve analysis under $\mathrm{I}-$ and $\mathrm{I}+$.

Further we examined 0,025\% 2-DG sensitivity using plate assay and growth curve analysis. We found reduced growth (growth curve and spot assay) was observed under I+ condition with the exposure of $0.025 \%$ 2-DG exposure in WT cells (Fig. 2B). The ume6 $\Delta$ still increased the growth was observed under I+ condition compared to the respective WT cells (Fig.2B).

A.

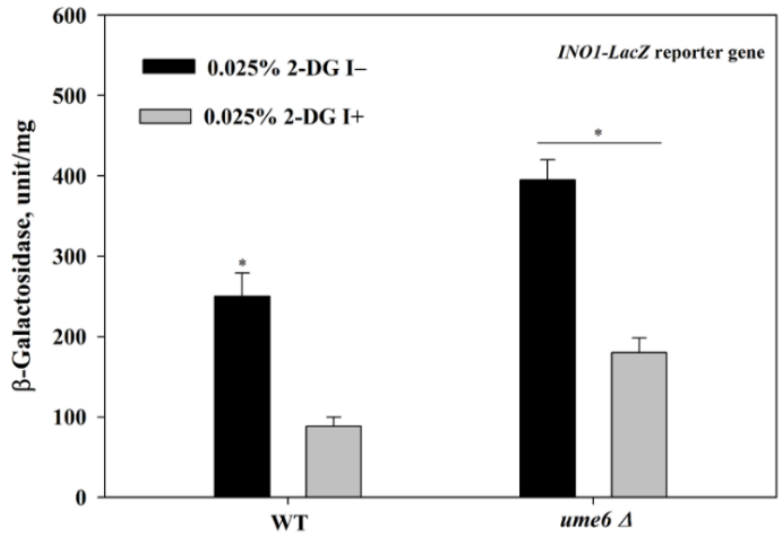

B

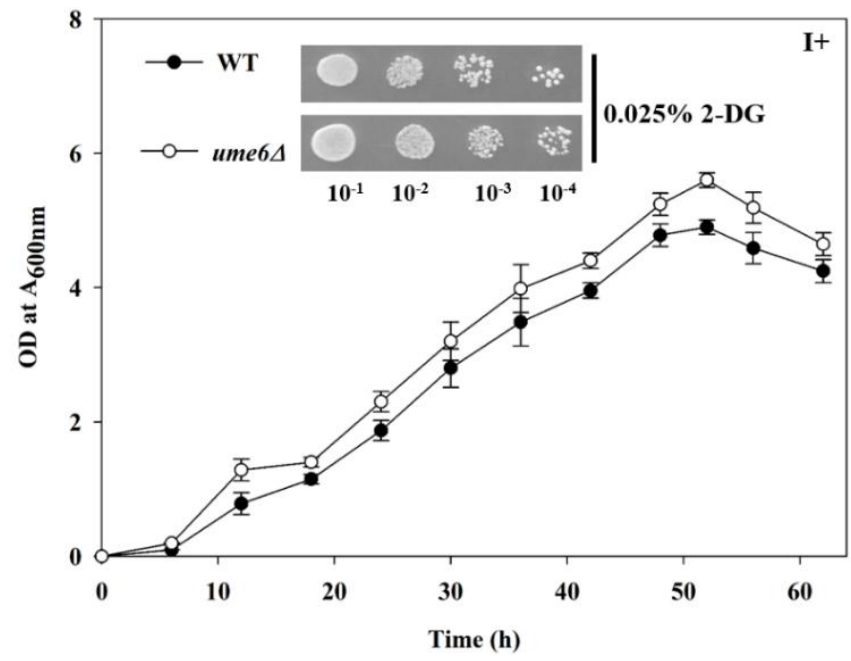

C

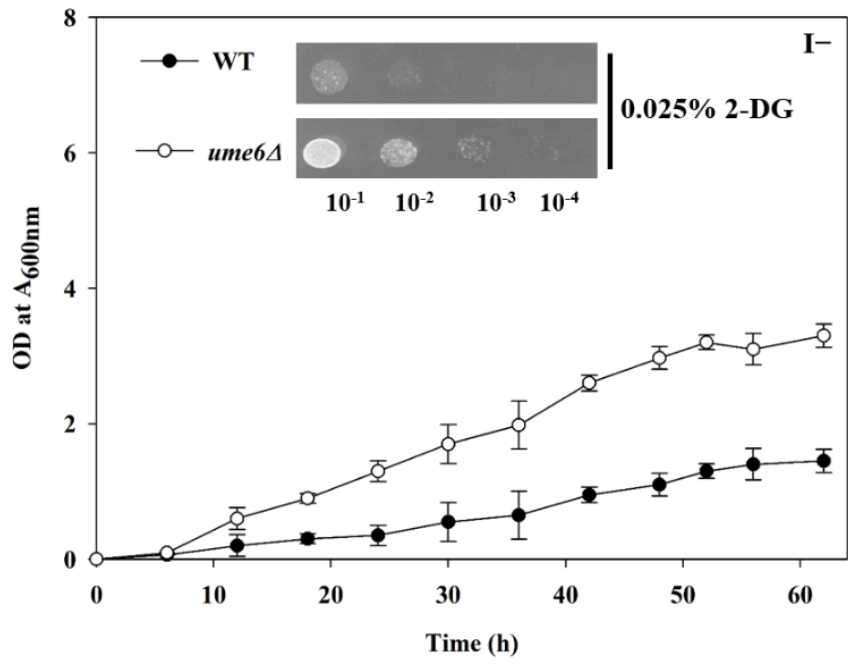

Fig.2 $\beta$-Galactosidase activity of INO1-LacZ and growth assessment under $0.025 \%$ of 2 -DG in WT and ume $\Delta \Delta$ cells 
under $\mathbf{I}-$ and $\mathbf{I}+$. (A). WT and ume6s strains harbouring YEp357R plasmid containing INOI-LacZ fusion gene and transformants were cultured into SD-U media up to mid log phase and the cells were washed and seeded in to the fresh media with the presence of $0.025 \% 2-\mathrm{DG}$ under $\mathrm{I}-$ and I+ cells. The cell extract were subjected to measure the $\beta$ Galactosidase activity. The specific $\beta$-Galactosidase activity was expressed as units mg-1 (nmol min-1 mg-1). (B and C) Spot test and growth curve analysis of WT and ume $6 \Delta$ strains under the exposure of $2-\mathrm{DG}(0.025 \%)$ in the presence and absence of inositol. The data shown is average of three independent experiments $(* \mathrm{P}<0.05)$.

The growth resistivity was supported for the increased $\beta$-galactosidase activity of INO1-LacZ in ume6 $\Delta$ cells under I+ condition compared to WT cells (Fig. 2B). Similarly, the deletion of OPII (Opi- phenotype) also rescue the growth with the exposure of 2-DG toxicity (10). Further, inositol limitation condition drastic growth reduction was observed in the exposure of $0.025 \%$ of $2-\mathrm{DG}$ exposure (Fig. 2C).

Effect of 0.025\% 2-DG on INO1-LacZ activity and toxicity assessment on plate assay and growth curve analysis under $\mathrm{I}-$ and $\mathrm{I}+$.

The INOI promoter activity and growth was measured in WT and ume $6 \Delta$ cells during the $2-\mathrm{DG}(0.05 \%)$ exposure under Iand $\mathrm{I}+$ condition. The presence of inositol the exposure of $0.05 \%$ 2-DG exposure observed a stringed growth in WT cells (Fig. 3B) compared to WT $0.025 \%$ 2-DG exposure (Fig.2 B). Further, the drastic reduction of INO1-LacZ activity was observed under I- condition, which indicates the increased concentration 2-DG highly toxic to the cells in WT cells under I- (Fig. 3A and C).

\section{A.}

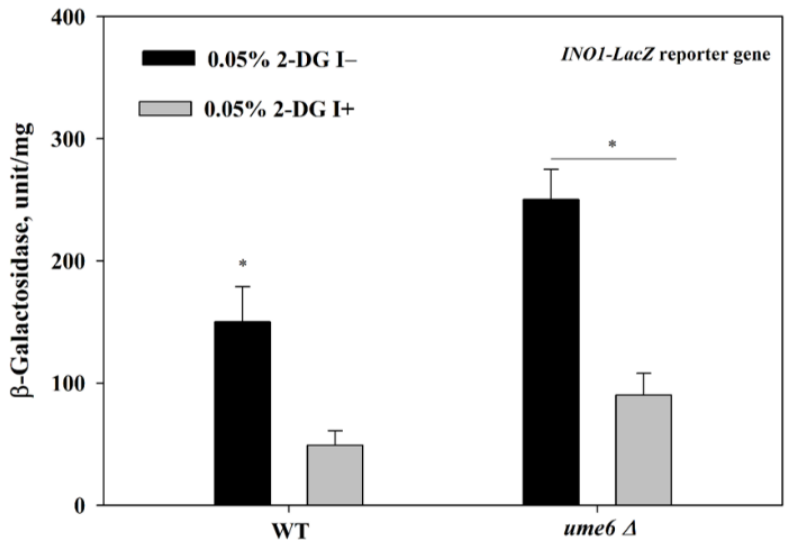

B.

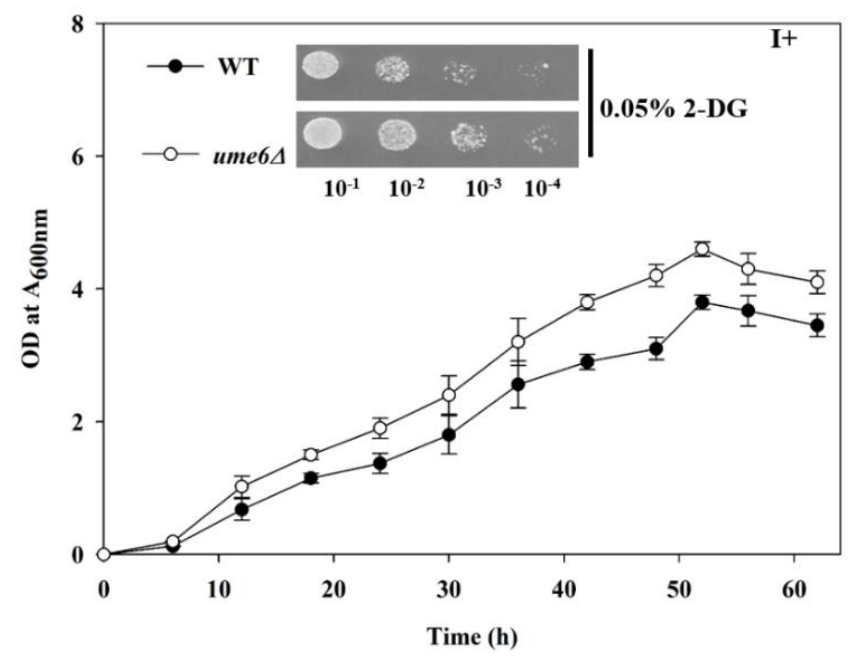

C.

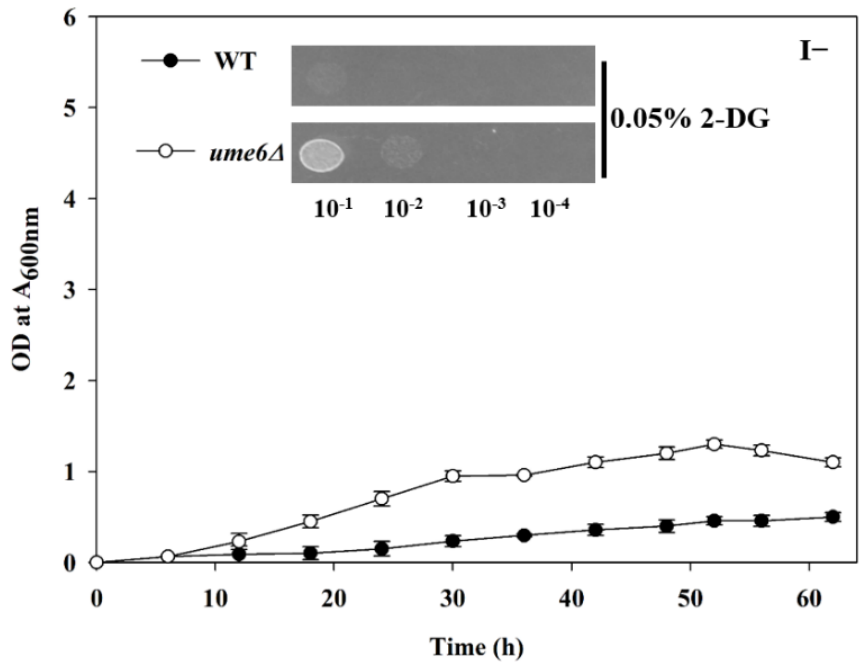

Fig.3. Effect of $0.05 \%$ 2-DG in $\beta$-Galactosidase activity of INO1-LacZ and growth assessment of WT and ume6 $\triangle$ cells under $\mathrm{I}-$ and $\mathrm{I}+$. (A).

WT and ume6s strains harbouring YEp357R plasmid containing INOI-LacZ fusion gene and transformants were cultured into SD-U media up to mid log phase and the cells were washed and seeded in to the fresh media with the presence of $0.025 \%$ 2-DG under I- and I+ cells. The cell extract were subjected to measure the $\beta$-Galactosidase activity. The specific $\beta$-Galactosidase activity was expressed as units mg-1 (nmol min-1 mg-1). (B) and (C). Spot test and growth curve analysis of WT and ume $6 \Delta$ strains under the exposure of $2-\mathrm{DG}(0.05 \%)$ in the presence and absence of inositol. The data shown is average of three independent experiments $(* \mathrm{P}<0.05)$.

On the other hand the inositol supplementation also reduced growth was observed in WT cell with the exposure of $0.05 \%$ 
2-DG. The deletion of UME6 still observed the growth in the exposure of $0.05 \%$ 2-DG under I- but not in WT cells, which depict the absence of inositol rescued the growth with the presence of $0.05 \%$ 2-DG (Fig. 3C). Finally the deletion of UME6 sustained the growth under $\mathrm{I}-$ with the presence of glucose analogue 2-DG toxicity.

\section{CONCLUSION.}

The exposure of glucose analogue 2-DG causes to reduces the growth and INOI-LacZ activity. Inositol was required for the growth under 2-DG toxicity. Hence the Ume6p negatively regulates the $I N O I$ transcription, even the deletion of UME6 increased the INOI transcription. The increased expression of INOI leads to rescued the growth under I- condition with the exposure of $2-\mathrm{DG}(0.025 \%$ and $0.05 \%$ ) in ume6 6 cells. Our study we conclude that the decreased INOI-LacZ leads to impaired the growth in the presence of 2-DG under I-. The inositol supplementation resistant to the 2-DG toxicity. Further work is need to find out the role of UME6 in 2-DG toxicity under inositol limitation condition.

\section{ACKNOWLEDGMENT}

We are thankful to DST-SERB Grant No: EMR/2016/001727, New Delhi under EMR scheme. We are grateful to Prof. Ram Rajasekharan (Central Food Technological Research Institute, Mysore, India) for providing yeast strains, over expression plasmids, reagents and instrument facility. We also thanks to John M. Lopes (College of Natural Sciences, University of Massachusetts, Amherst, MA) given that YEp357R-INO1-LacZ plasmid.

\section{REFERENCE}

[1]. $\quad$ K.S. Bowdish and A.P. Mitchell, "Bipartite Structure of an Early Meiotic Upstream Activation Sequence from Saccharomyces cerevisiae” Mol Cell Biol. Vol.13, Issue 4, pp 2172-2181, 1993.

[2]. J.C. Jackson and J.M. Lopes, "The yeast UME6 gene is required for both negative and positive transcriptional regulation of phospholipid biosynthetic gene expression". Nucleic Acids Res. Vol 24, Issue.7, pp 1322-1329, 1996.
[3]. S.A. Henry, S.D. Kohlwein and G.M. Carman, "Metabolism and regulation of glycerolipids in the yeast Saccharomyces cerevisiae”, Genetics. Vol.190, Issue.2, pp 317-349, 2013.

[4]. M.A. Hossain, J.M. Claggett, S.R. Edwards, A. Shi, S.L. Pennebaker, M.Y. Cheng, J. Hasty, T.L. Johnson, "Posttranscriptional Regulation of Gcrl Expression and Activity Is Crucial for Metabolic Adjustment in Response to Glucose Availability" Mol, Vol.62, Issue. 3, pp 346-358, 2016.

[5]. P. Biely, Z. Kratky, J. Kovařík, Š. Bauer, "Effect of 2Deoxyglucose on cell wall formation in Saccharomyces cerevisiae and its relation to cell growth inhibition", J Bacteriol, Vol.107, Issue.1, pp 121-129, 1971.

[6]. A.N. Wick, D.R. Drury, H.I. Nakada, J.B. Wolfe, "Localization of the primary metabolic block produced by 2deoxyglucose", J Biol Chem, Vol.224, Issue.2, pp 963-969, 1957.

[7]. W. Chen, M. Gueron, "The inhibition of bovine heart hexokinase by 2-deoxy-Dglucose-6-phosphate: Characterization by $31 P$ NMR and metabolic implications", Biochimie, Vol. 74, Issue.9-10, pp 867-873, 1992.

[8]. G.M. Carman and G.S. Han, "Regulation of phospholipid synthesis in the yeast Saccharomyces cerevisiae”, Annu. Rev. Biochem, Vol.80, pp 859-883, 2011.

[9]. S.A. Henry, S.D. Kohlwein and G.M. Carman, "Metabolism and regulation of glycerolipids in the yeast Saccharomyces cerevisiae”, Genetics. Vol.190, Issue.2, pp 317-349, 2013.

[10]. C. Ravi, V. Nachiappan, "In Saccharomyces cerevisiae the attenuation of the 2- deoxy-D-glucose toxicity is alleviated by inositol", International Journal of Scientific Research in Biological Sciences, Vol.5, Issue.6, pp.56-60, 2018.

[11]. L.E. Burton, W.W. Wells, "Studies on the effect of 5-thio-dglucose and 2-deoxy-d-glucose on myo-inositol metabolism", Archives of Biochemistry and Biophysics, Vol.181, Issue.2, pp 384-392, 1977.

[12]. M. Ralser, M.M. Wamelink, E.A. Struys, C. Joppich, S. Krobitsch, C. Jakobs, H. Lehrach, "A catabolic block does not sufficiently explain how 2-deoxy-D-glucose inhibits cell growth", Proc Natl Acad Sci USA, Vol.105, Issue.46, pp 17807-11, 2008

[13]. M.M. Bradford, "A rapid and sensitive method for the quantitation of microgram quantities of protein utilizing the principle of protein-dye binding”, Anal. Biochem, Vol.72, Issue.1-2, pp 248-254, 1976.

[14]. M. Rose and D. Botstein, "Construction and use of gene fusions to lacZ ( $\beta$ - galactosidase) that are expressed in yeast", Methods Enzymol, Vol.101, pp 167-180, 1983. 